\title{
GENDER DIFFERENCES AFTER DOWNHILL RUNNING FOR VOLUNTARY ISOMETRIC CONTRACTIONS OF KNEE EXTENSOR MUSCLES USING SURFACE EMG
}

\author{
Mark ET. Willems ${ }^{(A, B, C, D, F)}$, Simon R. Northcott ${ }^{(B, C, D, F)}$ \\ Faculty of Sport, Education \& Social Sciences, University of Chichester, United Kingdom
}

\begin{abstract}
Introduction: We examined gender differences for performance and surface electromyograms (sEMG) of knee extensors $1 \mathrm{hr}$ after downhill running.

Methods: Maximal voluntary isometric force (MVIF) and SEMG of vastus lateralis (VL) and vastus medialis (VM) muscles during $50 \% \mathrm{MVIF}$ were measured in 8 males $(22 \pm 4 \mathrm{yr}, 75.8 \pm 6.1 \mathrm{~kg}, 179.2 .0 \pm 5.0 \mathrm{~cm})$ and 8 females $(27 \pm 8 \mathrm{yr}$, $60.8 \pm 7.2 \mathrm{~kg}, 166.3 \pm 5.0 \mathrm{~cm})$ before and $1 \mathrm{hr}$ after downhill running $\left[\left(5 \times 8 \mathrm{~min},-12 \%, 60 \% \mathrm{~V}_{\text {max }}\right.\right.$ (females: $7.7 \pm 0.9 \mathrm{~km} \cdot \mathrm{h}^{-1}$, males: $\left.\left.10.4 \pm 0.5 \mathrm{~km} \cdot \mathrm{h}^{-1}\right)\right]$.

Results: Males had higher MVIF values $(674.3 \pm 59.8 \mathrm{~N}$ versus $480.6 \pm 49.9 \mathrm{~N}, P<0.001)$ and longer endurance times during $50 \%$ MVIF than females $(78.7 \pm 24.1 \mathrm{~s}$ versus $57.2 \pm 12.6 \mathrm{~s}, P<0.05)$ before downhill running. After downhill running, MVIF deficits were similar (males: $11.7 \pm 7.2 \%$, females: $7.5 \pm 7.7 \%$ ) with no changes in endurance times during $50 \% \mathrm{MVIF}$. After the downhill run, there was a trend in females, but not males, of an increased ratio for the root mean square (RMS) values of the VL and VM muscles during MVIF testing by $23 \%(P=0.054)$. After the downhill run, females, but not males, lowered during $50 \%$ MVIF the change in RMS for VM from $48 \pm 32 \%$ to $30 \pm 31 \%$ and for $\mathrm{VL}$ from $36 \pm 23 \%$ to $21 \pm 18 \%(P<0.05)$. For males, a trend was observed for the change in median frequency in VL during $50 \% \mathrm{MVIF}(P=0.05)$. After downhill running, females, altered the vastus medialis and vastus lateralis contributions to maximal isometric force production and the activity pattern of these muscles during submaximal isometric fatigue.

Conclusion: Acute neuromuscular adaptations after downhill running occur during voluntary isometric contractions of knee extensor muscles in females, but not in males.
\end{abstract}

Key words: Downhill running, skeletal muscle, electromyography, isometric contractions, muscle injury

\section{Introduction}

Following unaccustomed high-intensity muscle contractions, humans experience an immediate inability to produce maximal force. Such acute reductions in strength appear more common with eccentric rather than isometric or concentric contractions and the cause may be linked to injury rather than fatigue (1). The acute force loss by eccentric exercise is due to damage to force producing structures and excitationcontraction coupling failure $(2,3)$.

These mechanisms for muscle injury are also present following downhill running, an activity that is biased toward voluntary eccentric contractions of knee extensor muscles. After downhill running, acute force loss of knee extensor muscles occurred simultaneously with low-frequency fatigue (4), indicative of excitation-contraction coupling failure. In addition, extensive disorganization of the myofibrillar ultrastructure of the vastus lateralis muscle (5) combined with other indirect injury markers such as elevations of serum creatine kinase, myoglobin, troponin I, myosin heavy chain, plasma soluble intercellular adhesion molecule-1, collagen degrading enzymes and delayed onset muscle soreness are common observations after downhill running (6-11).
Apparently, alterations occur also in motoneuron pool excitability after downhill running (12). Others showed neuromuscular disturbances following eccentric exercise with elbow flexors $(13,14)$ and quadriceps femoris muscles (15). There is further evidence of selective damage of fast twitch muscle fibers after eccentric exercise in elbow flexors (16) and gastrocnemius muscle (17). For whole muscles, the amount of injury experienced by individual knee extensor muscles was different after eccentric knee extension exercise (18). Taken together these observations, if all or some of these events would occur immediately after downhill running, additional fatiguing exercise might be accomplished with neural adaptations due to muscle fiber type and muscle specific force losses by the eccentric contractions. For injured skeletal muscles that are part of a synergistic group such as the quadriceps femoris, i.e. the bi-articular rectus femoris and the mono-articular vastus medialis, vastus lateralis and vastus intermedius muscles, task redundancy in the system may allow performance of a submaximal task with different contributions from the individual muscles. Such task redundancy has been shown for knee extension exercise that was preceded by electrostimulated-fatigue of vastus lateralis (19). 
As far as we know, acute neural adaptations in damaged skeletal muscles following downhill running have not been studied. In the present study, neural adaptations after downhill running will be examined during sustained fatiguing isometric contractions using surface electromyography (sEMG). The surface electromyograms allow analysis of the signal amplitude in the time domain, quantified as the root mean square (RMS). RMS values are taken as an index of muscle activity as changes in this parameter during sustained fatiguing isometric contractions provide information on the recruitment of motor units and the mean firing rate of individual motor units (20). Surface electromyograms can also reveal the median frequency (MDF), a sEMG parameter that provides information on the power spectrum and shifts towards lower frequencies during sustained isometric contractions. Such shifts are taken as an index of muscle fatigue $(20,21)$.

Several studies examined the susceptibility for eccentric exercise between males and females in rats $(22,23)$ and humans $(4,24-26)$. Downhill running caused larger increases in serum levels of skeletal muscle proteins and enzymes in males than in females, probably due to an involvement of a larger muscle mass in males (10). However, animal and human studies reported no differences between acute maximal strength losses in males and females. Nevertheless, a difference in injury susceptibility could arise between males and females because of a difference in amount of slow and fast twitch muscle fibers $(27,28)$ that will not show up with maximal exercise. Any gender effect on muscle injury and neuromuscular adaptation as a result of downhill running might show up with submaximal exercise performed by synergistic muscles.

The purpose of this study was to determine the effect of gender on muscle performance of knee extensors after downhill running in young adults. It was hypothesized that after downhill running females and males would change the activation pattern of knee extensor muscles during maximal voluntary isometric contractions and sustained fatiguing isometric contractions.

\section{Methods}

Healthy female $(n=8$, mean \pm SD, age: $27 \pm 8 \mathrm{yr}$, body mass: $60.8 \pm 7.2 \mathrm{~kg}$, height: $166.3 \pm 5.0 \mathrm{~cm}$ ) and male university students ( $n=8$, age: $22 \pm 4 \mathrm{yr}$, body mass: $75.8 \pm 6.1 \mathrm{~kg}$, height: $179.2 .0 \pm 5.0 \mathrm{~cm}$ ) volunteered to participate in this investigation. Participants had no history of joint problems for knee and ankle. Female subjects were similar in age $(P=0.1)$, had lower body mass and were shorter than male subjects $(P<0.001)$. All experimental procedures were approved by the Ethics committee of the University of Chichester and performed according to the Declaration of Helsinki. Subjects were informed about the procedures, purposes and risks of the study and provided written informed consent prior to entering the study. All subjects were familiarized for performance of force measurements of the knee extensor muscles. A primary goal of the experiments was to determine whether there was an effect of gender on the force and surface electromyography of knee extensor muscles during maximal and submaximal voluntary contractions after a bout of downhill running. Each cycle of testing before and after downhill running consisted of 1 ) three warm-up contractions, 2) three maximal voluntary isometric contractions (MVIF), 3) a 50\%MVIF test to failure and 4) a MVIF 20 seconds after task failure. All mechanical recordings and surface electromyography were measured before and 1 hour after a bout of downhill running.

\section{Mechanical recordings}

Force recordings of knee extensor muscles of the non-dominant leg occurred with subjects seated in a custom-built chair with the upper body firmly restrained against the back of the chair using strap belts over chest and lower thigh. Both hip and knee joint angles were kept at $90^{\circ}$ (full extension is $0^{\circ}$ ). Participants had their arms crossed over the chest during all force testing. The ankle of the participant was connected with a chain to a calibrated stainless steel force transducer (model 616, Tedea-Huntleigh, Cardiff, UK) with a maximum of $2500 \mathrm{~N}$. Real-time force recorded by the force transducer was displayed using Chart for Windows (v. 3.4.1) on a computer screen positioned about $1.5 \mathrm{~m}$ in front of the subject, sampled and stored with a frequency of $1000 \mathrm{~Hz}$. Subjects performed three warm-up contractions (duration 4-6s) with a rest time of 1-min at an estimated intensity of about $30 \%$ of the force during maximal voluntary isometric contractions (MVIF), based on familiarization data. Then, subjects were instructed to perform three "all-out" maximal voluntary isometric contractions (MVIF) for about 2-5 $\mathrm{s}$ with a recovery time of 2 -min between trials. Participants were encouraged to exceed the maximum force value visible on the computer screen. Strong verbal encouragement was provided during MVIF (29). Following the MVIF, the monitor screen information for the Chart for Windows program was adjusted so that top and bottom of the screen represented 52.5\%MVIF and $47.5 \%$ MVIF, respectively. The target force for $50 \%$ MVIF was shown as a narrow horizontal bar in the middle of the screen. For the 50\%MVIF test, subjects were instructed to gradually increase the contraction force in order to minimize corrections needed in order to get the force signal on the screen after producing forces larger than 52.5\%MVIF. Subjects received strong verbal encouragement to keep the force signal visible within the screen for as long as possible. Each $50 \%$ MIVF was stopped by the experimenter when 
the force signal became lower than $2.5 \%$ of the target force for more than $2 \mathrm{~s}$. Our feedback protocol allowed subjects to produce force values close to 50\%MVIF. Before and after downhill running, mean force values over the total endurance time were $49.3 \pm 0.8 \% \mathrm{MVIF}$ and $50.0 \pm 1.2 \% \mathrm{MVIF}$ for males and $49.2 \pm 1.5 \% \mathrm{MVIF}$ and $49.3 \pm 1.2 \%$ MVIF for females. Twenty seconds on completion of the isometric fatigue task, subject performed one final "all-out" MVIF.

\section{Surface electromyography}

Bipolar surface EMG electrodes (Delsys, Boston, MA, USA), consisting of two parallel silver (99.9\%) bars, each $10 \mathrm{~mm}$ in length and $1.0 \mathrm{~mm}$ wide, with a interelectrode distance (center-to-center) of $10 \mathrm{~mm}$, were placed over the vastus medialis (VM) and vastus lateralis (VL) muscle of the non-dominant leg. The common-mode rejection ratio of the EMG electrodes is $<92 \mathrm{~dB}$. The reference electrode was placed over the patella. Before electrode placement, the skin was shaved, lightly abraded to reduce impedance between the electrode and cleansed with alcohol swabs. Electrodes were fastened to the skin with tape and location was traced with a marker pen to enable electrode placement at the same location in various sessions. Raw EMG signals were amplified $(\times 1000)$ per channel, sampled at a rate of $1024 \mathrm{~Hz}$ and stored on computer.

\section{Downhill running}

Subjects performed an incremental treadmill protocol on a Woodway Ergo ELG 70 (Cranlea and Co., Birmingham, UK) (gradient 1\%) with increases in speed of $1 \mathrm{~km} \cdot \mathrm{h}^{-1}$ every minute. The speed that could be maintained for a complete minute during the maximal test was taken as maximal speed $\left(\mathrm{V}_{\max }\right)$ (30). $\mathrm{V}_{\max }$ was $17.4 \pm 0.9 \mathrm{~km} \cdot \mathrm{h}^{-1}$ and $12.9 \pm 1.5 \mathrm{~km} \cdot \mathrm{h}^{-1}$ for males and females, respectively. Speed and gradient of the treadmill for the downhill run was set at $60 \% \mathrm{~V}_{\max }$ and $-12 \%$, respectively. Subjects ran 5 bouts of $8 \mathrm{~min}$ separated by a 2 min recovery (31) in which subjects remained standing on the non-moving part of the treadmill.

\section{Data analysis}

For each MVIF, the maximal force was quantified as the highest mean value over a $0.5 \mathrm{~s}$ period that included the peak force. The maximal force measured during any of the three trials was then taken as a measure of MVIF (i.e. MVIF ${ }_{\text {pre }}$ ). Fatigue induced by the sustained isometric contraction was quantified by the ratio (per cent) of the force value 20 seconds after completion of the isometric fatigue task to the maximal force value of the MVIF before the isometric fatigue task [(i.e. $100 \%$ - $\left.\left(\mathrm{MVIF}_{20 \mathrm{~s}} / \mathrm{MVIF}_{\mathrm{pre}} \times 100 \%\right)\right]$. Start of the endurance time was taken when the force signal was between $47.5 \%$ and $52.5 \%$ MVIF for at least $2 \mathrm{~s}$. The failure point was the time at which force fell below 47.5\% MVIF for longer than $2 \mathrm{~s}$. EMGworks software (Delsys, Boston, MA, USA) was used for bandpass filtering $(20-500 \mathrm{~Hz})$ of the raw EMG signals and calculation of the EMG parameters root mean square (RMS) and median frequency (MDF) for each muscle. The RMS and MDF values were taken as an index of muscle activity and muscle fatigue, respectively. For MVIF, the ratio of the RMS of the vastus lateralis muscle to the RMS of the vastus medialis muscle (i.e. $\mathrm{RMS}_{\mathrm{VL}} / \mathrm{RMS}_{\mathrm{VM}}$ ) was calculated. A change in this ratio would provide information on a change in contribution of these muscles to the maximal force producing capability of the knee extensor muscles after downhill running. EMG parameters for the 50\%MVIF (i.e. MDF and RMS) were calculated for successive sampling intervals for the first and last $10 \%$ of the duration time to task failure (i.e. endurance time) for the VL and VM muscles. The RMS of the EMG signal during the $50 \%$ MVIF was normalized with respect to the RMS values determined during the trail that provided maximal force. The percent change in RMS in the last 10\% time intervals (i.e. 90-100\%) from the first 10\% time interval (i.e. $0-10 \%$ ) and the absolute change in MDF in the last $10 \%$ time interval was calculated for each muscle before and after downhill running.

\section{Statistical analysis}

Unpaired student t-tests were used to test between males and females 1) MIVF before downhill running, 2) MVIF deficits after downhill running, 3) ratio of the RMS values of the vastus lateralis and vastus medialis muscles (i.e. $\mathrm{RMS}_{\mathrm{VL}}$ / $\mathrm{RMS}_{\mathrm{VM}}$ ) before downhill running, 4) a change in the ratio $\mathrm{RMS}_{\mathrm{VL}} / \mathrm{RMS}_{\mathrm{VM}}$ after downhill running, and 5) endurance times before downhill running, 6) the change in endurance times after downhill running, 7) the change in value of $100 \%$ - $\left(\mathrm{MVIF}_{20 \mathrm{~s}} / \mathrm{MVIF}_{\mathrm{pre}} \mathrm{x} 100 \%\right)$ (i.e. the force loss that was present 20 seconds after failure of the 50\%MVIF), 8) the percent change in RMS in the last $10 \%$ time interval for either vastus medialis and vastus lateralis, 9) MDF values between vastus lateralis and vastus medialis in the beginning of the 50\%MVIF, 10) the absolute change in MDF at the end of the 50\%MVIF for either vastus medialis and vastus lateralis. Data are presented as mean \pm SD. Significance was accepted at $P<0.05$.

\section{Results}

\section{Maximal voluntary isometric contractions} Maximal voluntary isometric force (MVIF)

Force of the knee extensor muscles of the female subjects $(480.6 \pm 49.9 \mathrm{~N}$, range from 419.8 to 561.0 $\mathrm{N})$ was $71 \%$ of the force value for the male subjects $(674.3 \pm 59.8 \mathrm{~N}$, range from 592.7 to $744.4 \mathrm{~N})(P<0.05)$ (Figure 1). Both female and male subjects showed a 
significant deficit in force of the knee extensor muscles $1 \mathrm{hr}$ after the downhill run (Figure 1), but the force deficits were similar (i.e. male: $11.7 \pm 7.2 \%$, range from 3.8 to $26.0 \%$, female: $7.5 \pm 7.7 \%$, range from -5.5 to $16.1 \%)$ suggesting that there was no gender effect on the susceptibility for maximal isometric force decline by muscle injury from a bout of downhill running.

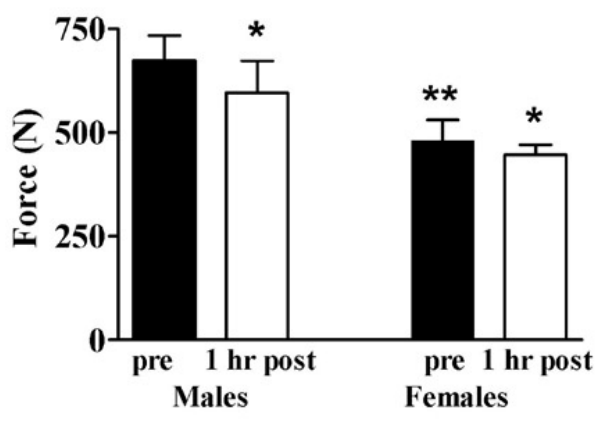

Fig. 1. Force (mean $\pm S D)$ of the knee extensor muscles of female and male subjects before and $1 \mathrm{hr}$ after downhill running (i.e. pre and $1 \mathrm{hr}$ post). * Significantly different from pre values for males and females, $P<0.05$. ${ }^{* *}$ Significantly different from pre value of males

\section{Surface electromyograms}

Before the downhill run, the ratio for the RMS values of the vastus lateralis (VL) and vastus medialis (VM) muscles (i.e. $\mathrm{RMS}_{\mathrm{VL}} / \mathrm{RMS}_{\mathrm{VM}}$ ) was similar for males $(0.49 \pm 0.25$, range from 0.19 to 1.04$)$ and females ( $0.44 \pm 0.08$, range from 0.33 to 0.54 ) (Figure 2). This suggests that for both males and females the contributions of VL and VM to voluntary maximal isometric force of the knee extensors were similar. However, 1 hr after the downhill, there was a trend for the ratio $\mathrm{RMS}_{\mathrm{VL}} / \mathrm{RMS}_{\mathrm{VM}}$ to be increased by $23 \%$ in female subjects (Figure 2) $(P=0.054)$ but it was not changed for the male subjects. This observation suggests that downhill running changed the contribution of the VL and VM to the task of producing maximal isometric force by the knee extensor muscles in females, but not in males.

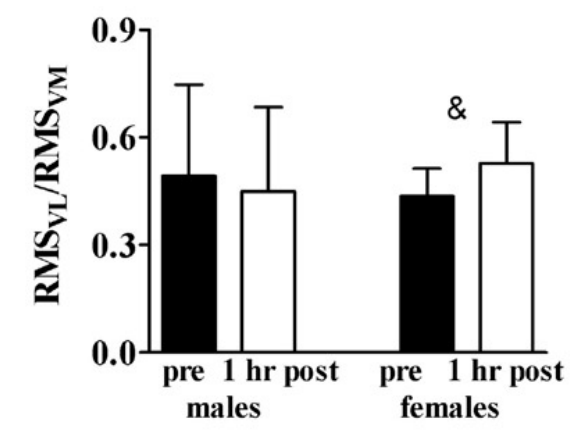

Fig. 2. Ratio of the RMS values of the vastus lateralis (VL) and vastus medialis (VM) muscles (i.e. $R M S_{V L} / R M S_{V M}$ ) for males and females during voluntary maximal isometric contractions (i.e. MVIF) before and $1 \mathrm{hr}$ after downhill running (i.e. pre and $1 \mathrm{hr}$ post). Data are presented as mean $\pm S D$. \&, trend to be different from pre values, $P=0.054$

\section{Submaximal isometric fatiguing contractions Endurance time}

The endurance time of knee extensor muscles during a sustained $50 \%$ MVIF was $55 \%$ higher for males (78.7 $\pm 24.1 \mathrm{~s}$, range: 46.2 to $113.3 \mathrm{~s}$ ) compared to females $(57.2 \pm 12.6 \mathrm{~s}$, range: 38.8 to 74.4$)(P<0.05)$. Endurance times in both males and females were not changed $1 \mathrm{hr}$ after the downhill run (males: $70.3 \pm$ $38.1 \mathrm{~s}$, range: 32.9 to $135.0 \mathrm{~s}$; females: $55.9 \pm 14.3 \mathrm{~s}$, range: 38.9 to $81.4 \mathrm{~s}$ ). The $50 \% \mathrm{MVIF}$ resulted in similar declines in the force 20 seconds after failure (i.e. male: $16.0 \pm 7.6 \%$, range: 6.9 to $28.2 \%$, female: $16.9 \pm 4.0 \%$, range: 9.7 to $21.2 \%$ ) with no changes for these declines when the 50\%MVIF test was performed $1 \mathrm{hr}$ after the downhill run (i.e. male: $13.4 \pm 5.5 \%$, range: 7.2 to $22.7 \%$, female: $17.7 \pm 5.2 \%$, range: 12.0 to $24.7 \%$ ).

\section{Electromyograms}

For the vastus medialis and vastus lateralis muscles of male subjects, the change in root mean square (RMS) during the sustained isometric contraction was not altered after the downhill run [(e.g. VM, before: $35.2 \pm 42.5 \%$, range: 2.0 to $132.2 \%$; after $44.1 \pm 42.0 \%$, range: -1.3 to $100.6 \%$ )] (Figure 3 ). In contrast, for the vastus medialis and vastus lateralis muscle of female subjects, the change in RMS was significantly lowered (VM: before $47.7 \pm 31.5 \%$, range: 11.1 to $114.1 \%$; after $30.1 \pm 31.2 \%$, range: 7.8 to $99.0 \%$; VL: before $35.5 \pm$ $22.7 \%$, range: 2.1 to $60.4 \%$; after $20.7 \pm 17.6 \%$, range: -0.6 to $49.1 \%)$ (Figure 3$)(\mathrm{P}<0.05)$.

At the beginning of the 50\%MVIF and before the downhill run, median frequency (MDF) values were higher in vastus lateralis muscle $(99 \pm 22 \mathrm{~Hz}$, range: 65 to $139 \mathrm{~Hz}$ ) compared to vastus medialis muscle $(74 \pm 7 \mathrm{~Hz}$, range: 66.6 to $81.2 \mathrm{~Hz}$ ) in male subjects $(\mathrm{P}=0.01)$. Such a difference was not present for the median frequency in female subjects [(VL: $72 \pm 13$ $\mathrm{Hz}$, range: 54 to $95 \mathrm{~Hz}$; VM: $70 \pm 7 \mathrm{~Hz}$, range: 61 to $82 \mathrm{~Hz}$ )]. Downhill running did not change the MDF

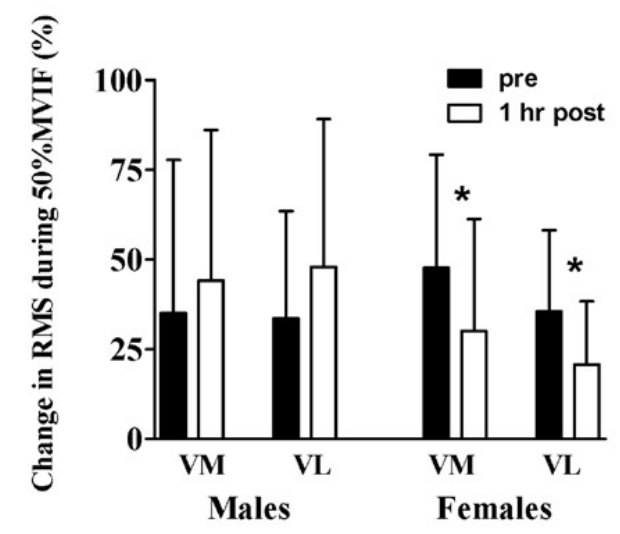

Fig. 3. Change in root mean square (RMS) during the sustained isometric contraction (50\%MVIF) for the vastus medialis and vastus lateralis muscles of male and female subjects before and after downhill running. Data are presented as mean $\pm S D$. ${ }^{*}$ Significantly different from pre values, $P<0.05$ 
value at the beginning of the 50\%MVIF in female and male subjects. For male subjects, there was a trend for a change in the shift of the MDF value of the vastus lateralis during a $50 \% \mathrm{MVIF}$ after downhill running (male: before $-17 \pm 11 \mathrm{~Hz}$, range: 1 to $-27 \mathrm{~Hz}$; after -10 $\pm 7 \mathrm{~Hz}$, range: 2 to $-19 \mathrm{~Hz})(\mathrm{P}=0.05)$. Such a shift was not observed in the vastus medialis of male subjects. For female subjects, there was no change in the shift of the MDF value of vastus medialis and vastus lateralis during a 50\%MVIF after downhill running (e.g. VL before: $-9 \pm 9 \mathrm{~Hz}$, range: 7 to $-25 \mathrm{~Hz}$; after $-10 \pm 6 \mathrm{~Hz}$, range: 0 to $-21 \mathrm{~Hz}$ ).

\section{Discussion}

The novel findings of the present study were that after a bout of mild downhill running females, but not males, alter the recruitment strategy of knee extensor muscles during voluntary efforts 1) to produce maximal isometric force and 2) to sustain a submaximal isometric force until failure. Evidence of neuromuscular adaptation was provided by the significant change in the ratio of the RMS values for the vastus lateralis and vastus medialis muscles. After downhill running, females, but not males, produced maximal isometric force of the knee extensor muscles with an increased and decreased contribution, respectively, of the vastus lateralis and vastus medialis muscles. In addition, females, but not males, performed the submaximal isometric fatigue task at 50\%MVIF with a reduced change in RMS values of the vastus medialis and vastus lateralis muscles. This alteration of recruitment strategy in females was not associated with an observable change in muscle fatigue (i.e. median frequency) in the vastii muscles. For males, the only observable change after the downhill run was the diminished ability to produce maximal isometric force (i.e. an isometric force deficit) and a trend for a smaller shift of the median frequency of the vastus lateralis muscle.

Isometric force deficits are considered to be the best measure of eccentric-contraction-induced muscle injury (32). Skeletal muscles from females and males suffer similar acute isometric force deficits from various types of eccentric exercise (e.g. 4,25,26) including downhill running (our study, males: 11.7\%, females: $7.5 \%$ ). Other downhill running studies reported isometric force deficits of $6 \%$ (33) and 22\% (31). Our force deficits were relatively mild compared to eccentric exercise in elbow flexor muscles for which deficits over $40 \%$ are not uncommon $(26,34,35)$. Part of the isometric force deficits may have been due to a depression in voluntary activation which was shown to occur after intermittent one-legged downhill running (4). However, intermittent one-legged downhill running for 30 minutes seemed to be more severe than our protocol and resulted in larger force deficits of $13.6 \%$ for males and $17 \%$ for females (4). The force deficits were most likely caused by muscle injury due to excitation-contraction coupling failure and damage to force producing structures. However, caution is needed when comparing potential mechanisms for force deficits from different studies because of differences in exercise intensity, exercise duration of the contractions, subject characteristics and muscle type. Nevertheless, both females and males in our study experienced the downhill run as an exercise of mild intensity and they did not report any subjective feelings of weakness 1 $\mathrm{hr}$ after the downhill run. If subjects had experienced some weakness from the downhill run, this may led to reduced endurance times during the $50 \% \mathrm{MVIF}$ which was not the case. Similarity in endurance times before and after downhill running was associated with similar changes in median frequency values during the $50 \%$ MVIF for both females and only for males a trend for smaller change in the vastus lateralis muscle. For males, the endurance time for knee extensor muscles to produce a $50 \%$ MVIF until failure was $78.7 \pm 24.1$ s. For studies in which males participated, large variation can be found for the average endurance time for knee extensor muscles during a 50\%MVIF: it ranges from values of $53 \pm 12 \mathrm{~s}$ to $120 \pm 21 \mathrm{~s}(33,36-42)$. We are aware of only one study that measured for females the endurance times of knee extensor muscles for $50 \%$ MVIF (endurance time $59 \pm 15 \mathrm{~s}, 40$ ) which was similar as the endurance time in our study (i.e. 57.2 $\pm 12.6 \mathrm{~s}$ ). Neither females or males showed a change in endurance times after the downhill run which is in contrast with Donnelly et al (33). A change from 62 to 55 seconds was observed after a downhill run of $45 \mathrm{~min}$, However, this measurement was taken 6 hours following the run (33). Constancy of endurance times for 50\%MVIF before and after downhill running may indicate that any contribution of total agonist and antagonist muscle activity towards maintaining a $50 \%$ MVIF was not changed.

After the downhill run, the MIVF was produced with a change in the ratio for the RMS values of the vastus lateralis and vastus medialis muscles (i.e. $\mathrm{RMS}_{\mathrm{VL}} / \mathrm{RMS}_{\mathrm{VM}}$ ) in females, but not in males. This observation suggests that the contribution of the vastus lateralis and vastus medialis muscles to the task of producing maximal isometric force with the knee extensor muscles was changed in females, but not in males. If we assume that the change in RMS values can be taken as an indication of motoneuron activation (43), females, but not males, reduce central and/or peripheral activation of motoneurons to the vastus medialis muscle after downhill running. Interestingly, females also performed the 50\%MVIF after the downhill run with reduced changes in activity of the vastus medialis and vastus lateralis muscle. Because group III and IV afferents are known to become activated following injury to muscle fibers, it is possible that 
these afferents from injured vastus medialis and vastus lateralis muscles in females may have reduced the descending drive to motoneurons (43), thus inhibiting spinal motoneurons responsible for activation of the vastii muscle. A recent study showed that eccentric contractions modulated central and peripheral fatigue during a $60 \mathrm{~s}$ maximal voluntary contraction 2 and 4 days after the exercise: central and peripheral fatigue progressed faster (44). A faster progress of central and peripheral fatigue in our study could have resulted in decreased endurance times but this was not the case. However, we need to emphasize again that our protocol was relatively mild in intensity. Interestingly, several studies have reported errors in the matching of force and position with elbow flexors immediately after eccentric exercise $(34,35,45)$. Such errors point to a disturbance in proprioception and indicate a change in motor control during submaximal force tasks. Any neuromuscular adaptation could be related to exercise-induced changes in afferent feedback from tendon organs (46) but not from muscle spindles because responses of muscle spindles during contractions were not changed after a series of eccentric contractions (47). Bulbulian et al (12) found evidence of reduced motoneuron pool excitability after downhill running by measuring the change in the ratio of the Hoffman reflex (H-wave) and the maximally electrically stimulated muscle action potential (M-wave) and after stimulation of the tibial nerve. However, we can only speculate at this point why such a muscle- and gender-specific neuromuscular adaptation occurred and cannot determine whether the changes occurred at the spinal or supraspinal level.

Differences in muscle activity during downhill running between males and females could have caused the adaptation in females. However, electromyographic studies have shown that preactivition for the quadriceps muscles during downhill running was similar for males and females (48). Similarity in preactivation, however, does not provide information on muscle activity during downhill running. Differences in kinematics between males and females during downhill running might make the females more susceptible for injury and therefore, potentially more damage is possible in specific muscles which warrant neuromuscular adaptation in females. However, many factors such as shoe characteristics influence the kinematic adaptations during running (49) and these were not controlled in our study. Interestingly, eccentric exercise with seated knee extension resulted in signs of greater muscle injury in rectus femoris (RF) compared to the vastii muscles based on magnetic resonance observations (18). These authors reasoned that restricted movement around the hip during seated knee eccentric extension exposed the rectus femoris to higher peak forces and displacements that would normally not be experienced during unrestricted movements (18). An increased susceptibility of the rectus femoris muscle for injury compared to the vastii muscles was suggested by Deschenes et al (15) based on an increase in integrated EMG of the rectus femoris muscle only after maximal concentric/eccentric knee exercise. Downhill running allows unrestricted movement of the vastii and rectus femoris muscles and differences in kinematics during downhill between females and males could have led to variation in the degree of injury experienced by the individual knee extensor muscles. Small differences in muscle specific fiber length changes during eccentric contractions and eccentric forces for individual knee extensor muscles during downhill running may have been unfavourable for one or more of the knee extensor muscles of females. Subjects were not instructed regarding their control of stride length during the downhill run. We can only speculate at this point why the females would change neuromuscular strategy to perform maximal and submaximal isometric contractions after downhill running. Possibly, muscle-specific injury requires compensation by other muscles (i.e. rectus femoris and vastus obliquus) of the knee extensor group to perform maximal and submaximal isometric contractions. Such compensation by synergistic muscles is not uncommon. For example, compensation by vastus medialis and rectus femoris during fatiguing submaximal knee extension has been shown when the exercise was preceded by electrostimulated-fatigue of vastus lateralis only (19). Thus, acute modifications of neuromuscular coordination among synergistic muscles allow to perform the exercise during muscle-specific fatigue (19). Our monitoring of only vastus lateralis and vastus medialis does not allow to pinpoint which of the knee extensor muscles may have been more injury susceptible, if this was the case at all. The neuromuscular adaptation in females might not be even linked to the degree of muscle injury resulting from the downhill running. Fast-twitch muscle fibers are thought to be susceptible for eccentric contraction-induced injury than slow-twitch muscle fibers (50) and differences in fiber type distributions in individual knee extensor muscles between males and females were reported $(28,51)$ but were not found by others (52). However, there exist large variations in fiber type distributions in males and females and taking into account that our sample size was relatively small, it would make a speculative discussion of a possible effect of fiber type. Furthermore, no relationship exists between endurance time for a $50 \% \mathrm{MVIF}$ and myosin heavy chain composition of vastus lateralis muscle (42) and the similarity in median frequency during the 50\%MVIF before and after downhill running suggested that there was no specific effect on fast twitch muscle fibers. This is in contrast with eccentric exercise of elbow flexors for which lower 
median frequency values were recorded $1 \mathrm{hr}$ after the exercise (16). Further studies should examine whether neuromuscular adaptations would occur in males and females with downhill running protocols of more severe intensity and for isometric fatigue tasks at force levels lower and higher than 50\%MVC.

\section{Conclusion}

It is concluded that after downhill running females but not males change the neural drive to vastus medialis and vastus lateralis muscle during submaximal isometric contractions. It may be possible that disproportionate muscle injury in females might have required a change in recruitment strategy to produce voluntary isometric contractions. Mild injury to synergistic muscles in females caused by eccentric contractions may alter the recruitment patterns of individual muscles during voluntary isometric contractions.

\section{Acknowlegdements}

We thank John Kelly (University of Chichester) and Dr. Craig Sale (Nottingham Trent University) for statistical support and helpful discussions regarding the findings.

\section{References}

1. McNeil CJ, Allman BL, Symons TB, et al. Torque loss induced by repetitive maximal eccentric contractions is marginally influenced by work-to-rest ratio. Eur J Appl Physiol 2004; 91: 579-85.

2. Proske U, Morgan DL. Muscle damage from eccentric exercise: mechanism, mechanical signs, adaptation and clinical applications. J Physiol 2001; 537: 333-45.

3. Warren GL, Ingalls CP, Lowe DA, et al. What mechanisms contribute to the strength loss that occurs during and in the recovery from skeletal muscle injury? J Orthop Sports Phys Ther 2002; 32: 58-64.

4. Martin V, Millet GY, Martin A, et al. Assessment of low-frequency fatigue with two methods of electrical stimulation. $J$ Appl Physiol 2004; 97: 1923-9.

5. Féasson L, Stockholm D, Freyssenet D, et al. Molecular adaptations of neuromuscular disease-associated proteins in response to eccentric exercise in human skeletal muscle. $J$ Physiol 2002; 543: 297-306.

6. Akimoto T, Furudate M, Saitoh M, et al. Increased plasma concentrations of intercellular adhesion molecule-1 after strenuous exercise associated with muscle damage. Eur J Appl Physiol 2002; 86: 185-90.

7. Byrnes WC, Clarkson PM, White JS, et al. Delayed onset muscle soreness following repeated bouts of downhill running. J Appl Physiol 1985; 59: 710-5.

8. Kalinski MI. Characterization of biochemical and immunological responses to various types of exercise linked to muscle injury. Med Sport 2007; 11(1): 1-6.

9. Koskinen SOA, Höyhtya M, Turpeenniemi-Hujanen T, et al. Serum concentrations of collagen degrading enzymes and their inhibitors after downhill running. Scand J Med Sci Sports 2001; 11: 9-15.

10. Sorichter S, Mair J, Koller A, et al. Release of muscle proteins after downhill running in male and female subjects. Scand J Med Sci Sports 2001; 11: 28-32.

11. Yu JG, Malm C, Thornell LE. Eccentric contractions leading to DOMS do not cause loss of desmin nor fibre necrosis in human muscle. Histochem Cell Biol 2002; 118: 29-34.
12. Bulbulian R, Bowles DK. Effect of downhill running on motoneuron pool excitability. J Appl Physiol 1992; 73: 968-73.

13. Bottas R, Linnamo V, Nicol C, et al. Repeated maximal eccentric actions causes long-lasting disturbances in movement control. Eur J Appl Physiol 2005; 94: 62-9.

14. Miles MP, Ives JC, Vincent KR. Neuromuscular control following maximal eccentric exercise. Eur J Appl Physiol Occup Physiol 1997; 76: 368-74.

15. Deschenes MR, Brewer RE, Bush JA, et al. Neuromuscular disturbance outlasts other symptoms of exercise-induced muscle damage. J Neurol Sci 2000; 174: 92-9.

16. Felici F, Colace L, Sbriccoli P. Surface EMG modifications after eccentric exercise. J Electromyogr Kinesiol 1997; 7: 193-202.

17. Jones DA, Newham DJ, Round JM, et al. Experimental human muscle damage: morphological changes in relation to other indices of damage. J Physiol 1986; 375: 435-48.

18. Prior BM, Jayaraman RC, Reid RW, et al. Biarticular and monoarticular muscle activation and injury in human quadriceps muscle. Eur J Appl Physiol 2001; 85: 185-90.

19. Akima H, Foley JM, Prior BM, et al. Vastus lateralis fatigue alters recruitment of musculus quadriceps femoris in humans. J Appl Physiol 2002; 92: 679-84.

20. De Luca CJ. The use of surface electromyography in biomechanics. J Appl Biom 1997; 13: 135-63.

21. Mannion AF, Dolan P. Relationship between myoelectric and mechanical manifestations of fatigue in the quadriceps femoris muscle group. Eur J Appl Physiol Occup Physiol 1996; 74: 411-19.

22. Komulainen J, Koskinen SOA, Kalliokoski R, et al. Gender differences in skeletal muscle fibre damage after eccentrically biased downhill running in rats. Acta Physiol Scand 1999; 165: 57-63.

23. Willems MET, Stauber WT. Force deficits after repeated stretches of activated skeletal muscles in female and male rats. Acta Physiol Scand 2001; 172: 63-7.

24. Dannecker EA, Hausenblas HA, Kaminski TW, et al. Sex differences in delayed onset muscle pain. Clin J Pain 2005; 21: $120-6$.

25. Rinard J, Clarkson PM, Smith LL, et al. Response of males and females to high-force eccentric exercise. J Sports Sci 2000; 18: 229-36.

26. Sayers SP, Clarkson PM. Force recovery after eccentric exercise in males and females. Eur J Appl Physiol 2001; 84: 122-6.

27. Komi PV, Karlsson J. Skeletal muscle fibre types, enzyme activities and physical performance in young males and females. Acta Physiol Scand 1978; 103: 210-18.

28. Simoneau JA, Bouchard C. Human variation in skeletal muscle fiber-type proportion and enzyme activities. Am J Physiol 1989; 257: E567-72.

29. McNair PJ, Depledge J, Brettkelly M, et al. Verbal encouragement: effects on maximum effort voluntary muscle action. $\mathrm{Br}$ J Sports Med 1996; 30: 243-5.

30. Noakes TD, Myburgh KH, Schall R. Peak treadmill running velocity during the $\mathrm{VO} 2$ max test predicts running performance. J Sports Sci 1990; 8: 35-45.

31. Eston RG, Lemmey AB, McHugh P, et al. Effect of stride length on symptoms of exercise-induced muscle damage during a repeated bout of downhill running. Scand J Med Sci Sports 2000; 10: 199-204.

32. Warren GL, Lowe DA, Armstrong RB. Measurement tools used in the study of eccentric contraction-induced injury. Sports Med 1999; 27: 43-59.

33. Donnelly AE, Maughan RJ, Whiting PH. Effects of ibuprofen on exercise-induced muscle soreness and indices of muscle damage. Br J Sports Med 1990; 24: 191-5.

34. Saxton JM, Clarkson PM, James R, et al. Neuromuscular dysfunction following eccentric exercise. Med Sci Sports Exerc 1995; 27: 1185-93.

35. Proske U, Gregory JE, Morgan DL, et al. Force matching errors following eccentric exercise. Hum Mov Sci 2004; 23: 365-78.

36. de Ruiter CJ, Elzinga MJH, Verdijk PWL, et al. Voluntary drive-dependent changes in vastus lateralis motor unit firing 
rates during a sustained isometric contraction at $50 \%$ of maximum knee extension force. Pflugers Arch 2004; 447: 436-44.

37. Ebenbichler GR, Kollmitzer J, Glöckler L, et al. The role of the biarticular agonist and cocontracting antagonist pair in isometric muscle fatigue. Muscle Nerve 1998; 12: 1706-13.

38. Kalmar JM, Cafarelli E. Effects of caffeine on neuromuscular function. J Appl Physiol 1999; 87: 801-8.

39. Mad'setti $O$, Guevel A, Legros $P$, et al. Prediction of endurance capacity of quadriceps muscles in humans using surface electromyogram spectrum analysis during submaximal voluntary isometric contractions. Eur J Appl Physiol 2002; 87: 509-19.

40. Maughan RJ, Harmon M, Leiper JB, et al. Endurance capacity of untrained males and females in isometric and dynamic muscular contractions. Eur J Appl Physiol Occup Physiol 1986; 55: 395-400.

41. Masuda K, Masuda T, Sadoyama T, et al. Changes in surface EMG parameters during static and dynamic fatiguing contractions. J Electromyogr Kinesiol 1999; 9: 39-46.

42. Taylor AD, Humphries B, Smith P, et al. Electrophoretic separation of myosin heavy chain isoforms in the human $\mathrm{m}$. vastus lateralis: references to reproducibility and relationships with force, electromechanical delay, fibre conduction velocity, endurance and electromyography. Arch Physiol Biochem 1997; 105: 10-8.

43. Gandevia SC. Spinal and supraspinal factors in human muscle fatigue. Physiol Rev 2001; 81: 1725-89.

44. Endoh T, Nakajima T, Sakamoto M, et al. Effects of muscle damage induced by eccentric exercise on muscle fatigue. Med Sci Sports Exerc 2005; 37: 1151-56.

45. Walsh LD, Allen TJ, Gandevia SC, et al. Effect of eccentric exercise on position sense at the human forearm in different postures. J Appl Physiol 2006; 100: 1109-16.

46. Gregory JE, Morgan DL, Proske U. Tendon organs as monitors of muscle damage from eccentric contractions. Exp Brain Res 2003; 151: 346-55.

47. Gregory JE, Morgan DL, Proske U. Responses of muscle spindles following a series of eccentric contractions. Exp Brain Res 2004; 157: 234-40.
48. DeMont RG, Lephart SM. Effect of sex on preactivation of the gastrocnemius and hamstring muscles. Br J Sports Med 2004; 38: 120-4.

49. Hardin EC, van den Bogert AJ, Hamill J. Kinematic adaptations during running: effects of footwear, surface, and duration. Med Sci Sports Exerc 2004; 36: 838-44.

50. Fridén J, Sjöström M, Ekblom B. Myofibrillar damage following intense eccentric exercise in man. Int J Sports Med 1983; 4: $170-6$.

51. Simoneau JA, Lortie G, Boulay MR, et al. Skeletal muscle histochemical and biochemical characteristics in sedentary male and female subjects. Can J Physiol Pharmacol 1985; 63: 30-5.

52. Staron RS, Hagerman FC, Hikida RS, et al. Fiber type composition of the vastus lateralis muscle of young men and women. J Histochem Cytochem 2000; 48: 623-9.

Received: December 22, 2008

Accepted: February 05, 2009

Published: February 10, 2009

Address for correspondence:

Mark Willems

Faculty of Sport, Education \& Social Sciences,

University of Chichester, College Lane Chichester,

West Sussex, PO19 6PE, United Kingdom

fax: + $44(0) 1243816080$

e-mail: m.willems@chi.ac.uk

Simon R. Northcott: s.northcott@chi.ac.uk

\begin{tabular}{llll}
\hline Authors' contribution & B - Data Collection & D - Data Interpretation & F - Literature Search \\
A - Study Design & C - Statistical Analysis & E - Manuscript Preparation & G - Funds Collection
\end{tabular}

